Research Journal of Medical Sciences 4 (2): 98-101, 2010

ISSN: $1815-9346$

(C) Medwell Journals, 2010

\title{
An Interesting Unusual Case Report of Madly Endodontic Treatments
}

\author{
C. Stavrianos, A. Eliades and A. Kokkas \\ Department of Endodontics, School of Dentistry, Aristotle University, Thessaloniki, Greece
}

\begin{abstract}
A number of reports have been published concerning neuralgia and other complications of the inferior alveolar nerve following a penetration of root canal filling or endo-file into or close to the mandibular canal. Endodontic infections of posterior maxillary teeth sometimes spread to the maxillary sinus, generating severe complications. Also, endodontic implications of the maxillary sinus include the introduction of endodontic instruments and materials beyond the apices of posterior teeth in close proximity to the sinus. Clinicians should be aware of the fact that endodontic instruments and filling materials (solid or liquid) can be extended in such a degree that can lead to neurological or sinus complications, i.e., dysaesthesia of the inferior alveolar nerve or sinusitis, due to the proximity of the apices of the mandibular molars and premolars to the inferior alveolar canal and the maxillary posterior teeth to the sinus floor membrane. An interesting and unusual case of iatrogenic complications due to madly endodontic therapies is presented.
\end{abstract}

Key words: Endodontic therapies, iatrogenic complications, endodontic files, over-extension of gutta-percha cone, chronic apical periodontitis

\section{INTRODUCTION}

The final objective of endodontic treatment is to provide hermetic obturation of the root canal system with an inert, biocompatible and dimensionally stable filling material according to a satisfactory obturation of the root canal. Ideally, the filling material and the endodontic instruments should be limited to the root canal without extending to periapical tissues or other neighbouring structures (Poveda et al., 2006). Filling material, broken file, gutta-percha extruded in the periapical area causes a foreign reaction of the connective tissue (Kafas et al., 2009). Depending on the organism's immune, the connective tissue tends to absorb the foreign body or more frequently, surround it with a fibrous capsule.

A number of reports have been published concerning neuralgia and other complications of the inferior alveolar nerve following a penetration of root canal filling or endo-file into or close to the mandibular canal (Allantar et al., 1994; Orstravic et al., 1983). Also, hypaesthesia is the most frequently encountered complication of over-extension endodontic treatment instrument (Zeigler and Serene, 1984) and/or filling material if these are in contact or close to the inferior alveolar canal, such as they can cause injury of the inferior alveolar nerve.

One of the most seen iatrogenic complications in endodontics is overfilling of the root canal which has a negative effect on prognosis for endodontically treated teeth (Brkić et al., 2009). More than a half of the overfilled teeth heal satisfactorily after proper endodontic therapy but in cases of injury of the inferior alveolar nerve or presence of filling material in soft tissues or sinus spaces a surgical approach is necessary (Brkić et al., 2009).

Endodontic infections of posterior maxillary teeth sometimes spread to the maxillary sinus, generating severe complications.

Endodontic implications of the maxillary sinus include extension of periapical infections into the sinus, the introduction of endodontic instruments and materials beyond the apices of posterior teeth in close proximity to the sinus and the risks and complications associated with endodontic surgery (Hauman et al., 2002). In this study a case of iatrogenic complications due to madly endodontic therapies is presented.

\section{MATERIALS AND METHODS}

Case report: A 52-year-old Mediterranean male was referred to the School of Dentistry of Aristotle University of Thessaloniki in September of 2009. The patient reported that he has been to a dentist 2 years ago and he was aware that he had dental problems, since then because of sensitive teeth and loose tooth. Earlier episodes of sensitivity were recalled in the area of the right and left mandible and sometimes in the maxilla. Furthermore the

Corresponding Author: Christos Stavrianos, Department of Endodontics, School of Dentistry, Aristotle University, Thessaloniki, Greece 
patient reported that some day before he had awakened with severe pain in the left mandible area. He also stated that certain swelling and pain disappeared after taking antibiotic and analgesic by his own initiative. When questioned for his late seek of dental assistance, the patient neglect to report these thinking they were un-important symptoms which would pass soon.

The patient's medical history was remarkable and local factors such as jaw fractures, facial trauma, third molar surgery, odontogenic cyst, local tumor infiltration and metastasis of cancer, all of which have been proven to be responsible for such symptoms were eliminated. No allergies were reported.

In the clinical examination, there was no lympadenopathy and any swelling in the area prescribed wasn't detected. The intraoral examination showed carious right mandibular 1 st molar and 2nd left mandibular molar. Also in the maxilla, the 3rd molars were found carious too. Vital pulp examination on these teeth revealed necrosis of them. The electronic pulp tester did not elicit a response from these teeth whereas all the other teeth in the area responded within normal limits.

The patient was evaluated radiographically by panoramic radiograph on this visit (Fig. 1). The radiographic examination was remarkable. In the upper jaw, the 3rd molars were found carious. A deep carious involvement of the left 3rd maxillary molar near the pulp chamber was found. The right 3rd maxillary molar was depicted carious too but with a broken endodontic file in the mesial-buccal root (Fig. 2). The broken instrument was showed to traverse the whole root (from the pulp chamber to the apex) and be exerted from the apical foramen of the tooth to the periapical tissue for distance of $1-2 \mathrm{~mm}$. There were no signs of trauma to the sinus floor membrane and no symptoms of possible sinusitis according to the patient history. In the mandible, the left 2nd molar was decayed and with a fractured amalgam filling in the crown.

The distal root of the tooth was found to be traversed by a gutta-percha cone (Fig. 3) exerted from the apex. The over-extension of the root canal cone into the periapical tissue was for at least $6 \mathrm{~mm}$ long and after a pigtag shaped curve it ends at the top of the corresponding alveolar ridge. The mesial root was found un-treated and a periapical radiolucency was presented to be large enough and extended. According to radiographic and clinical findings the diagnose was easily excluded to be an chronic apical periodontitis. The repeat of the endodontic therapy with the combination of surgical endodontic is decided. On the other side the 1st mandibular right molar was carious and fractured too and was found to be

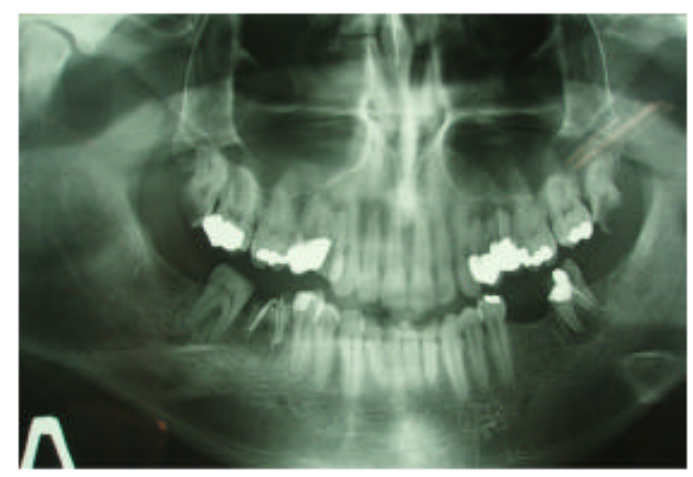

Fig. 1: Panoramic radiography of the patient on the first visit shows three cases of madly endodontic therapies

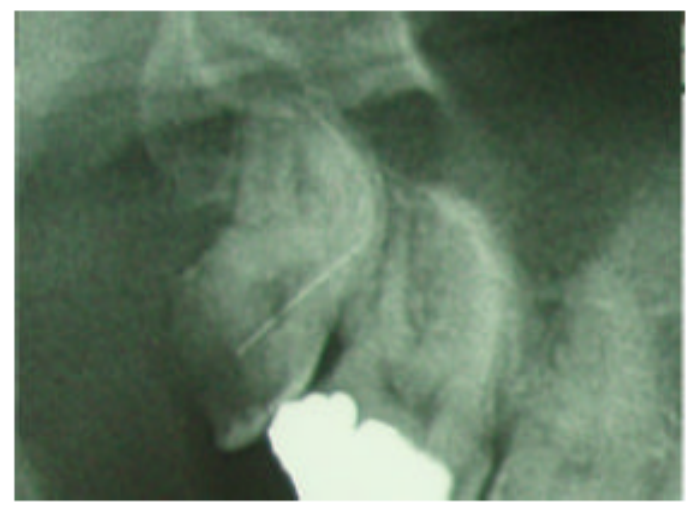

Fig. 2: Besides of the carious activity this part of the panoramic radiography shows a broken endo-file in the mesial root of the 3rd maxillary molar which is overextended for a short distance of $1 \mathrm{~mm}$ to the periapical tissue. This complication could be an impact on the sinus floor membrane and a causal of sinusitis

endodontically treated. The mesial-buccal root canal was overfilled and an extrusion of endodontic filling material was presented (Fig. 4). Also, the distal root was treated too but the endodontic paste or gutta-percha cone did not reach-fill the entire root canal length. The radiography didn't show any radiolucency in the periapical areas of the roots. The repeat of endodontic treatment (conventionally) is decided to be a safe prognostic therapy.

The patient was in formed for the madly endodontic therapies while he was not from his dentist. The radiographic and clinical examination as well as the recorded data enable us to diagnose the patient's condition which will be discussed in the discussion. 


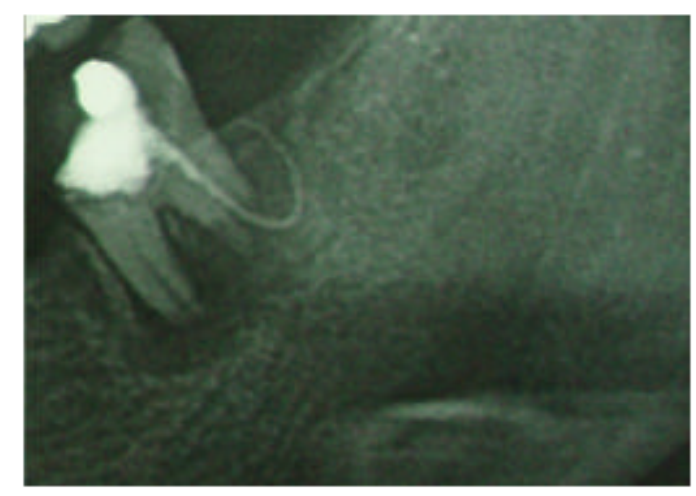

Fig. 3: The distal root of the mandibular 3rd molar was found to be traversed by a gutta-percha cone which is exerted from the apex. The overextension of the root canal cone into the periapical tissue $w$ as for at least $6 \mathrm{~mm}$ long and after a pig-tag shaped curve it ends at the top of the corresponding alveolar ridge. The mesial root of the tooth was found "un-treated" and a periapical radiolucency was presented to be large enough and extended which indicated a chronic apical periodontitis

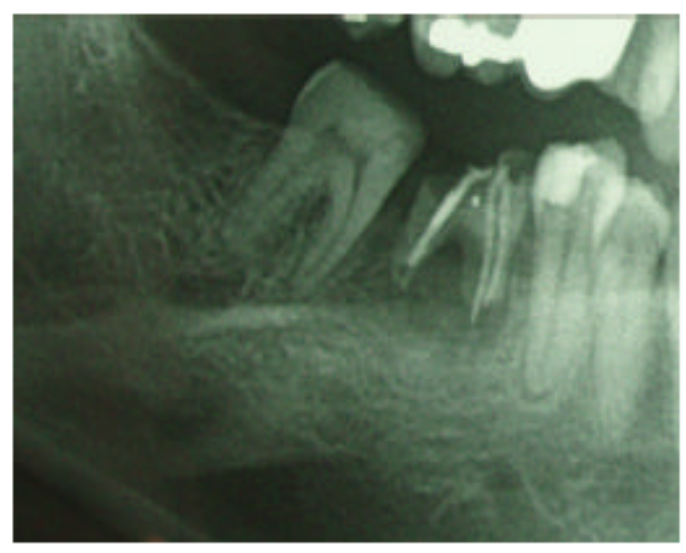

Fig. 4: The 1 st mandibular right mol ar $w$ as found carious and fractured and madly endodontically treated. The mesial-buccal root canal was overfilled and an extrusion of endodontic filling material was presented. Also, the distal root was treated too but the endodontic paste or gutta-percha cone did not reach-fill the entire root canal length. The radiog aphy didn't show any radiolucency in the periapical areas of the roots

\section{RE SULTS AND DISCU SSION}

In regard to filler pastes and cements (Alantar ef al., 1994), 4 routes are described from the periapical zone: toward the mandibular canal, systemic diffusion through a periapical vein, drainage through lymphatic vessels and progression toward soft tissues between bone and mucosal membrane. The case reported with the mandible left 2 nd molar corresponds with the 4 th of these routes.

Overfilling the root canal caries some risk, the greatest risk of which appears to be damage to the mandibular nerve with a resultant paresthesia or anesthesia of its sensory distribution (Barkhordar and N guyen, 1985). Several factors may be related to loss of sensitivity. Procechual errors such as overinstrumentation and an overextended filling may result in traum to the nerve. The filling material may also damage the nerve chemically according to its toxicity. Prolonged compression or chemical effects may internupt the blood supply, resulting in necrosis of the surrounding tissue (periapical tissue necrosis). The degree of damage depends in part on the biocompatibility of the filling material.

Sometimes severe complications, due to endodontic etiolog can affect the maxillary sinus. Endodontic implications of the maxillary sinus include extension of periapical infections into the simus, the introduction of endodontic instruments and materials beyond the apices of posterior teeth in close proximity to the sinus (Hauman et al., 2002).

The study of Nimigean et al. (2006) present the various problems encountered during endodontic treatment of posterior maxillary teeth. About 125 cases of odontogenic chronic m axillary simusitis were reviewed retrospectively. Chroric apical periodontitis was the cause in 99 cases and traumatising endodontic treatment in 26 cases. Foreign intrasinusal bodies were occasionally seen as a consequence of different endodontic treatments of posterior maxillary teeth

In the case described above, the overextension of fillingmaterial from the apical foramen of the mesial-buccal root canal ( 1 st mandibular right molar) did not show any damage of the periapical tissues, according to the normal bone density of the area shown from the radiographic ex amination. On the other side, the over-extension of the gutta-percha cone from the distal root apex of the 2nd mandibular left m olar would be dangerous to be a causal factor of sensory alteration in the left mandibular region with the result of a paresthesia of the mandibular nerve (nerve compression). A1so, the periapical infection of the mesial root of the molar could be responsible too for a mandibular nerve compression ( $Y$ eler ef al., 2004). In the maxilla the 3rd molars, al so would be causal factors of an acute or chronic inflammation of the corresponding sinus (simusitis). On the left side, an acute apical periodontitis due to the necrosis of the pulp would be responsible for 
the sinusitis. On the other side, the broken endo-file could be a mechanical and a septic causal factor for the occurrence of a acute sinusitis.

\section{CONCLUSION}

A knowledge of dento-antral relationships is particularly important in the prevention of sinusal accidents and mandibular nerve paresthesia during various therapeutic manoeuvres which should be performed according to and depending on the regional morphology. The anatomical and clinical significance of the inferior alveolar canal and maxillary sinus in relation to conventional and surgical endodontic therapy is considered. Mechanical and chemical effects may have contributed to the development of these iatrogenic complications. Clinicians should be aware of the fact that endodontic instruments and filling materials (solid or liquid) can be extended in such a degree that can lead to neurological or sinus complications, i.e. dysaesthesia of the inferior alveolar nerve or sinusitis, due to the proximity of the apices of the mandibular molars and premolars to the inferior alveolar canal and the maxillary posterior teeth to the sinus floor membrane.

\section{REFERENCES}

Allantar, A., H. Tarragano and B. Levevre, 1994. Extrusion of endodontic filling material into the insertions of the mylohyoid muscle. Oral Surg. Oral Med. Oral Pathol., 78: 646-649.

Barkhordar, R. and N. Nguyen, 1985. Paresthesia of the mental nerve after overextension with $\mathrm{AH} 26$ and gutta-percha: Report of case. J. Am. Dental Assoc., 110: 202-203.
Brkić, A., B. Gürkan-Köseoglu and V. Olgac, 2009. Surgical approach to iatrogenic complications of endodontic therapy: A report of 2 cases. Oral Surg. Oral Med. Oral Pathol. Oral Radiol. Endod., 107: $50-53$.

Hauman, C.H., N.P. Chandler and D.C. Tong, 2002. Endodontic implications of the maxillary sinus: A review. Int. Endod. J., 35: 127-141.

Kafas, P., T. Upile, N. Angouridakis, C. Stavrianos, N. Dabarakis and W. Jerjes, 2009. Dysaesthesia in the mental nerve distribution triggered by a foreign body: A case report. Cases J., 2: 169-169.

Nimigean, V.R., V. Nimigean, N. Maru, D. Andressakis, D.G. Balatsouras and V. Danielidis, 2006. The maxillary sinus and its endodontic implications: Clinical study and review. B-ENT, 2: 167-175.

Orstravic, D., P. Brodin and E. Ass, 1983. Paresthesia following endodontic treatment: Survey of the literature and report of a case. Int. Endod. J., 16: 167-172.

Poveda, R., V.J. Bagán, J.M.D. Fernández and J.M.M. Sanchis, 2006. Mental nerve paresthesia associated with endodontic paste within the mandibular canal: Report of a case. Oral Surg. Oral Med. Oral Pathol. Oral Radiol. Endod., 102: 46-49.

Yeler, H., I. Ozeç and E. Kiliç, 2004. Infection-related inferior alveolar and mental nerve paresthesia: Case reports. Quintessence Int, 35: 313-316.

Zeigler, P.E. and T.P. Serene, 1984. Failure in Therapy. In: Pathways of the Pulp, Cohen, S. and R.C. Burns (Eds.). 3rd Edn., CV Mosby Co., St Louis, pp: 786-819. 